\title{
Influence of magnetic field and magnetic anisotropy on the quench characteristics of $\mathrm{Bi}-2223 / \mathrm{Ag}$ multifilamentary tapes
}

\section{AUTHOR(S):}

Nakamura, T; Tsuchiya, S; Fujio, A; Hoshino, T; Muta, I

\section{CITATION:}

Nakamura, T ... [et al]. Influence of magnetic field and magnetic anisotropy on the quench characteristics of Bi-2223/Ag multifilamentary tapes. IEEE TRANSACTIONS ON APPLIED SUPERCONDUCTIVITY 2001, 11(1): 3341-3344

\section{ISSUE DATE:}

2001-03

URL:

http://hdl.handle.net/2433/39943

\section{RIGHT:}

(c)2001 IEEE. Personal use of this material is permitted. However, permission to reprint/republish this material for advertising or promotional purposes or for creating new collective works for resale or redistribution to servers or lists, or to reuse any copyrighted component of this work in other works must be obtained from the IEEE. 


\title{
Influence of Magnetic Field and Magnetic Anisotropy on the Quench Characteristics of Bi-2223/Ag Multifilamentary Tapes
}

\author{
Taketsune Nakamura, Shingo Tsuchiya, Akihisa Fujio, Tsutomu Hoshino and Itsuya Muta
}

\begin{abstract}
We examined the normal propagation characteristics of Bi-2223/Ag multifilamentary tapes as a function of magnetic field direction. Anisotropic current transport characteristics were estimated by taking into account the effect of the misorientation of the grains. Anisotropic normal zone propagation properties were examined based on the numerical simulation, and showed that the thermal properties were influenced by the anisotropic current transport characteristics.
\end{abstract}

Keywords--Bi-2223, Anisotropy, Normal Zone Propagation Properties

\section{INTRODUCTION}

$\mathrm{T}$ REMENDOUS efforts have been made for the development of the power application systems using high- $T_{c}$ superconducting (HTS) tapes. Silver sheathed $\mathrm{Bi}_{2} \mathrm{Sr}_{2} \mathrm{Ca}_{2} \mathrm{Cu}_{3} \mathrm{O}_{10+x}(\mathrm{Bi}-2223 / \mathrm{Ag})$ tape is the most promising candidate for such applications. For the design of the HTS systems, the estimation of the stability is crucial. However, HTS is thermally stable at elevated temperatures. Furthermore, current transport curves of HTS are complicated depending on temperature and/or transport current, and are also anisotropic with respect to the applied field direction. This means that the Joule heating process is complicated. Therefore, taking account of such effects is important to the quantitative understanding of the thermal properties. Shimizu et al.[1] showed that the power-law expression for the $E-J$ curves in zero magnetic field can be used for the calculation of the normal zone propagation properties. On the other hand, Kiss et al.[2,3] proposed the analytical expression for the properties in high magnetic fields, and Okamoto et al.[4] showed that the $E-J$ curves of $\mathrm{Bi}-2223 / \mathrm{Ag}$ multifilamentary tapes can be described quantitatively by that expression. Therefore, appropriate expression for the $E-J$ characteristics has to be selected depending upon the application, i.e., zerofield or non-zero field application. On the other hand, we showed that the angular dependence of the current transport characteristics in $\mathrm{Bi}-2223 / \mathrm{Ag}$ multifilamentary tape is well described by the model which takes into account the misorientation of the grains [5].

In this paper, we present the normal propagation properties of the Bi-2223/Ag multifilamentary tape as a function of temperature and magnetic field direction studied by computer simulation.

T. Nakamura, S. Tsuchiya, A. Fujio, T. Hoshino and I. Muta are with the Graduate School of Engineering, Kyoto University, Kyoto 606-8501, Japan. E-mail: tk_naka@kuee.kyoto-u.ac.jp

\section{EXPRESSION FOR $E-J$ CURVES}

A. E $-J$ curves in zero external field

$E-J$ characteristics in zero external field are expressed by the power-law approximation with index, $n$, as follows:

$$
E=E_{\mathrm{co}}\left(\frac{J}{J_{\mathrm{c} 0}}\right)^{n}
$$

where $E_{\mathrm{c} 0}$ is electric field criterion to define $J_{\mathrm{c} 0}$. As shown in Fig.2, this expression is valid in a wide range of temperatures.

\section{B. $E-J$ curves in high external field}

$E-J$ properties in high magnetic field are very complicated, depending upon the temperarure and/or current. We use the expression proposed by Kiss et al.[2,3]. In this expression, so-called glass-liquid transition temperature, $T_{\mathrm{g}}$, is introduced, and $E-J$ properties are expressed as follows:

for $T \leq T_{\mathrm{g}}$;

$$
E(J, T) \cong \frac{\rho_{\mathrm{ff}}}{m+1} J\left[\frac{J}{J_{0}(T)}\right]^{m}\left[1-\frac{J_{\mathrm{cm}}(T)}{J}\right]^{m+1}
$$

for $T>T_{\mathrm{g}}$

$$
\begin{aligned}
E(J, T) \cong \frac{\rho_{\mathrm{ff}}}{m+1}\left|J_{\mathrm{cm}}(T)\right|\left[\frac{\left|J_{\mathrm{cm}}(T)\right|}{J_{0}(T)}\right]^{m} & \\
\cdot & {\left[\left(1+\frac{J}{\left|J_{\mathrm{cm}}(T)\right|}\right)^{m+1}-1\right] }
\end{aligned}
$$

where $\rho_{\mathrm{ff}}$ and $m$ are, respectively, the uniform flux flow resistivity and the parameter describing the shape of the local critical current distribution. $J_{\mathrm{cm}}$ is a minimum local critical current density, and expressed as follows:

$$
J_{\mathrm{cm}}=J_{\mathrm{T}}\left|1-\frac{T}{T_{\mathrm{g}}}\right|^{2 \nu}
$$

where $J_{\mathrm{T}}$ and $\nu$ are constant. $J_{0}\left(T^{\prime}\right)$ is roughly proportional to $T^{-1}$.

\section{Anisotropy of $E-J$ curves}

We have taken account of the misorientation of the grains in $\mathrm{Bi}-2223 / \mathrm{Ag}$ tape, and shown that the glass-liquid transition flux density, $B_{\mathrm{g}}$, as a function of the angle between the external field direction and the axis perpendicular to 
the tape surface, $\varphi$, is well described by the following expression[5]:

$$
B_{\mathrm{g}}(\varphi, T)=B_{\mathrm{g}, \perp}(T)\left(\cos ^{2} \varphi+\gamma^{-2} \sin ^{2} \varphi\right)^{-\frac{1}{2}}
$$

where $\gamma$ is the effective anisotropy factor and defined as follows:

$$
\gamma=\frac{B_{\mathrm{g}, / /}}{B_{\mathrm{g}, \perp}}
$$

$B_{\mathrm{g}, / /}$ and $B_{\mathrm{g}, \perp}$ are $B_{\mathrm{g}}$ for, respectively, parallel and perpendicular field direction with respect to the tape face. We assume that (5) and (6) are valid for arbitrary temperature considered in this paper.

\section{Simulation Detail}

Fig.1 shows the shematics of the simulation model. The total length of $100 \mathrm{~mm}$ is considered, and the $50 \mathrm{~mm}$ of it is simulated as both sides are symmetrical. For simplicity, Bi$2223 / \mathrm{Ag}$ multifilamentary tape is treated as two layers of superconducting Bi layer and silver sheath. The following heat balance equation is solved by finite difference method:

$$
c(T) \frac{\partial \theta}{\partial t}=k\left(\frac{\partial^{2} \theta}{\partial x^{2}}+\frac{\partial^{2} \theta}{\partial y^{2}}\right)+G+Q_{\mathrm{d}}
$$

where $c$ and $k$ are, respectively, the heat capacity and the thermal conductivity. $\theta$ is temperature difference and defined as $T-T_{0}$ with bias temperature $\left(T_{0}\right) . G(=E J)$ is Joule heating and calculated with one of (1), (2) and (3). $Q_{\mathrm{d}}$ is disturbance which is applied by the heater, considered on the silver sheath as shown in Fig.1. We take into account the temparature dependence of $c$. Parameter $k$ is assumed to be constant. Conduction cooling is considered, and boundary condition for the surface of the tape is adiabatic. The temperature of the end of the tape is fixed at $T_{0}$. Furthermore, we assume that the equipotential surface through any points is at right angles to the longitudinal direction of the tape at that points. Then, current density flowing in the superconducting $\mathrm{Bi}$ layer, $J_{\mathrm{sc}}$, is determined with the ratio of silver, $\eta$, as follows:

$$
\frac{\eta}{\eta+1} \frac{E\left(J_{\mathrm{sc}}\right)}{\rho_{\mathrm{ag}}}=J_{\text {total }}-\frac{1}{\eta+1} J_{\mathrm{sc}}
$$

where $J_{\text {total }}$ and $\rho_{\text {ag }}$ are the total transport current and the resistivity of the silver sheath, respectively. Equation (8) is calculated numerically using Newton-Raphson method.

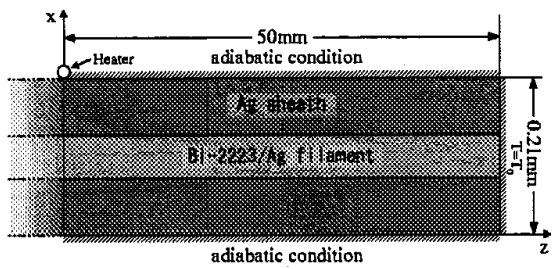

Fig, 1. Simulation Model.

\section{Results and Discussion}

\section{A. Normal Propagation Properties in Zero Magnetic Field}

Firstly, we simulate the normal propagation properties in zero magnetic field by using the measured $V-I$ curves. $\mathrm{Bi}-2223 / \mathrm{Ag}$ tape, which parameters are listed in TABLE I, was cooled by a GM cryocooler. $V-I$ measurements were performed by means of the DC four points method.

Fig. 2 shows the temperature dependence of $V-I_{\text {sc }}$ characteristics in zero magnetic field. Effect of the sharing current flowing in the silver sheath was eliminated with the measured voltage, $V$, by $I_{\mathrm{sc}}=I_{\text {total }}-V / R_{\text {ag }} . R_{\text {ag }}$ is the resistance of the silver sheath. As can be seen in Fig.2, we can roughly describe the $V-I_{\mathrm{sc}}$ curves with (1) in a wide range of temperature. Fig. 3 shows the temperature dependence of the critical currents and the $n$-values which are defined by the voltage criterion of $0.6 \mu \mathrm{V}$, corresponding to the electric field criterion of $1 \mu \mathrm{Vcm}^{-1}$. We can describe $I_{\mathrm{c}}$ with the following expression:

$$
I_{\mathrm{c}}(T)=I_{\mathrm{c} 0}\left(1-\frac{T}{T_{\mathrm{c}}}\right)^{\alpha}
$$

Critical temperature, $T_{\mathrm{c}}(=107.6 \mathrm{~K})$, was obtained from the Resistance vs. Temperature relation measured with small bias current $(=20 \mathrm{~mA}) . \alpha(=1.39)$ is a fitting parameter. We can see that the $n$-value is temperature dependent weakly at $T \leq 95 \mathrm{~K}$, and decreases drastically above the temperature. Then this dependency is also taken into account for the simulation.

TABLE I

The Parameters of the Sample used for the $V-I$ MEASUREMENTS IN ZERO MAGNETIC FIELD

\begin{tabular}{cc}
\hline Cross sectional area & $3.2 \mathrm{~mm}^{w} \times 0.21 \mathrm{~mm}^{t}$ \\
Number of filaments & 55 \\
Ratio of silver & 2.8 \\
$T_{\mathrm{c}}$ & $107.6 \mathrm{~K}$ \\
\hline
\end{tabular}

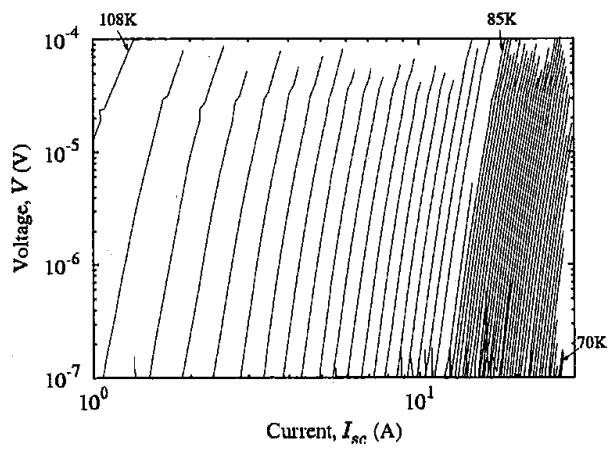

Fig. 2. Temperature dependence of $V-I_{3 c}$ curves in zero magnetic field. The sharing current flowing in the silver $\operatorname{sheath}\left(V / R_{\mathrm{ag}}\right)$ is eliminated by $I_{s c}=I_{\text {total }}-V / R_{\mathrm{ag}}$. Temperature intervals are $0.4 \mathrm{~K}$ from $70.0 \mathrm{~K}$ to $85.0 \mathrm{~K}$, and $1.0 \mathrm{~K}$ from $85.0 \mathrm{~K}$ to $108.0 \mathrm{~K}$, respectively. 


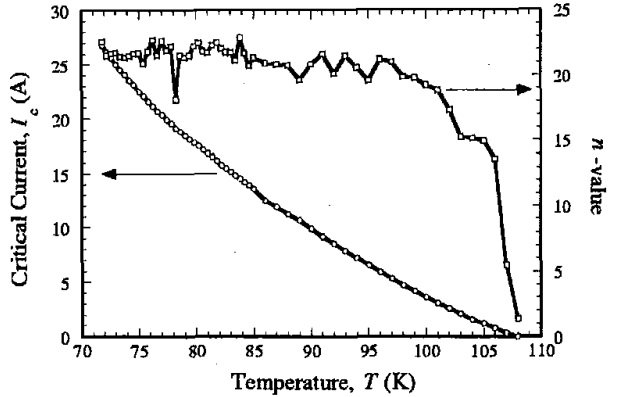

Fig. 3. Temperature dependence of $I_{\mathrm{c}}$ and $n$-value in $\mathrm{Bi}-2223 / \mathrm{Ag}$ multifilamentary tape in zero external field. $I_{c}$ and $n$-value are defined with electric field criterion of $1 \mu \mathrm{Vcm}^{-1}$.

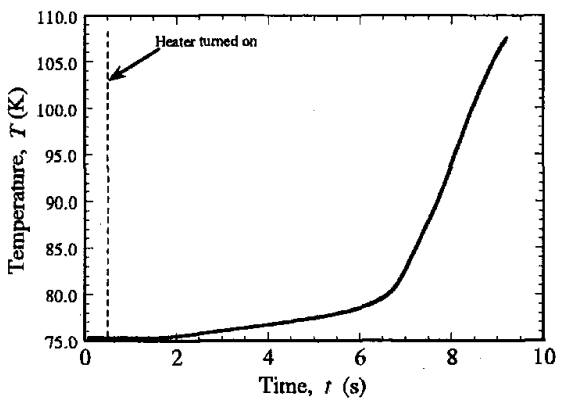

Fig. 4. Temperature trace with a transport current of $19.0 \mathrm{~A}$, $I_{\text {total }} / I_{\mathrm{c}}=0.9$. Initial temperature is $75.3 \mathrm{~K}$. Distance from the heater is $6.0 \mathrm{~mm}$. Heater(heat flux of $1.6 \times 10^{5} \mathrm{Wm}^{-2}$ ) is turned on at $t=1.0 \mathrm{~s}$.

Combining (1), (7), (8) and (9), one can simulate the normal zone propagation properties. Fig. 4 shows temperature traces for different heater powers . Bias current, 19.0A, is applied to the sample. After the temperature is stabilized, the heater (heat flux of $1.6 \times 10^{5} \mathrm{Wm}^{-2}$ ) is turned on from $t=1.0$ to $15.0 \mathrm{~s}$. By turning on the heater, temperature begins to increase gradually due to the Joule heating in the Bi filaments. Then, current sharing is increased due to the rising temperature, and temperature increases rapidly at $t=15 \mathrm{~s}$. This behavior is qualitatively in agreement with the experimental data in [6].

\section{B. Normal Propagation Properties in high magnetic field}

Next, we simulate the normal propagation properties in high magnetic field with the same simulation code in subsection IV-A. In high magnetic field, $V-I_{\mathrm{sc}}$ characteristics vary complicatedly depending upon the temperature and/or the transport current, and (1) is no more applicable. Then, we adopt (2) and (3) for the calculation of the Joule heating. The experimental data in high magnetic field is not obtained yet, hence we use the data measured in a field of $1 \mathrm{~T}$ by Okamoto et al.[4]. Fig.5 shows the $E-J$ curves reproduced by (2) and (3), and the parameters obtained in [4] are listed in TABLE II. Furthermore, in order to study the influence of the anisotropic current transport

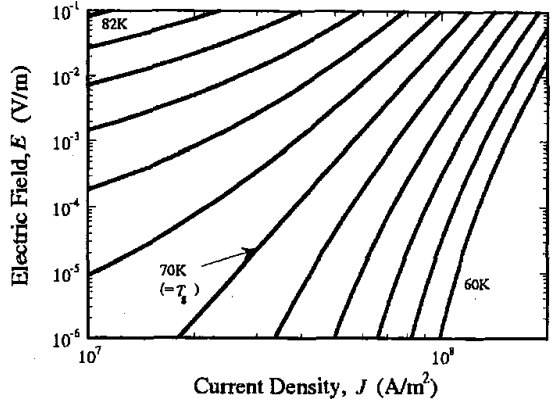

Fig. 5. Temperature dependence of $E-J$ characteristics reproduced by (2) and (3) with the parameters listed in TABLE II. External magnetic field $(1 \mathrm{~T})$ is applied perpendicularly to the sample face $[4]$.

TABLE II

The Parameters of THE eXPerimanetal data used For the SIMULATION IN HIGH MAGNETIC FIELD[4]

\begin{tabular}{cc}
\hline$m$ & 5.1 \\
$\nu$ & 0.5 \\
$\rho_{\mathrm{ff}}$ & $1 \times 10^{-7} \Omega \mathrm{m}$ \\
$J_{\mathrm{T}}$ & $5.15 \times 10^{8} \mathrm{~A} / \mathrm{m}^{2}$ \\
$T_{\mathrm{g}}$ at $1 \mathrm{~T}$ & $70.0 \mathrm{~K}$ \\
$B_{\mathrm{g}, / /} / B_{\mathrm{g}, \perp}$ & 5.0 \\
$T_{\mathrm{c}}$ & $101 \mathrm{~K}$ \\
\hline
\end{tabular}

characteristics, (5) and (6) are assumed to be valid for (2), (3) and (4). Temperature dependence of glass-liquid transition flux density for perpendicular field direction, $B_{\mathrm{g}, \perp}$, is expressed from [4] as follows:

$$
B_{\mathrm{g}, \perp}=B_{\mathrm{g}, \perp}(0)\left(1-\frac{T}{T_{\mathrm{c}}}\right)^{\beta}
$$

where $B_{\mathrm{g}, \perp}(0)=5.9 \mathrm{~T}, T_{\mathrm{c}}=101 \mathrm{~K}$, and $\beta=1.5$, respectively. Combining (5) and (10), and replacing parameters from $B_{\mathrm{g}}$ to $B$ and $T$ to $T_{\mathrm{g}}$, one can estimate the angular dependent $T_{\mathrm{g}}$ as follows:

$$
\begin{aligned}
& T_{\mathrm{g}}(\varphi, B) \\
= & {\left[1-\left(\frac{B}{B_{\mathrm{g}, \perp}(0)}\right)^{\frac{1}{\beta}}\left(\cos ^{2} \varphi+\gamma^{-2} \sin ^{2} \varphi\right)^{\frac{1}{2 \beta}}\right] T_{\mathrm{c}} }
\end{aligned}
$$

Fig.6 shows $T_{\mathrm{g}}$ vs. $\varphi$ curves calculated by (11). $E-J_{\mathrm{sc}}$ curves at temperature below and above $T_{\mathrm{g}}(\varphi)$ are determined by (2) and (3), respectively. Angular dependence of the normal propagation properties are considered by varying $T_{\mathrm{g}}$ with (11). Fig.7 and 8 show the time evolutions of the voltages and temperatures for different field directions. Thermal disturbance(heat flux of $1.8 \times 10^{5} \mathrm{Wm}^{-2}$ ) is applied by the beater from $t=1.0$ to $15 \mathrm{~s}$ with the bias current of $15.0 \mathrm{~A}$. Initial temperature is $70.0 \mathrm{~K}$ which corresponds to $T_{\mathrm{g}, \perp}\left(=T_{\mathrm{g}}\left(\varphi=0^{\circ}\right)\right)$ in this field $(1.0 \mathrm{~T})$. That is, the properties except at $\varphi=0^{\circ}$ are at temperatures below $T_{\mathrm{g}}(\varphi)$ before turning on the heater, and $E-J_{\mathrm{sc}}$ properties are 


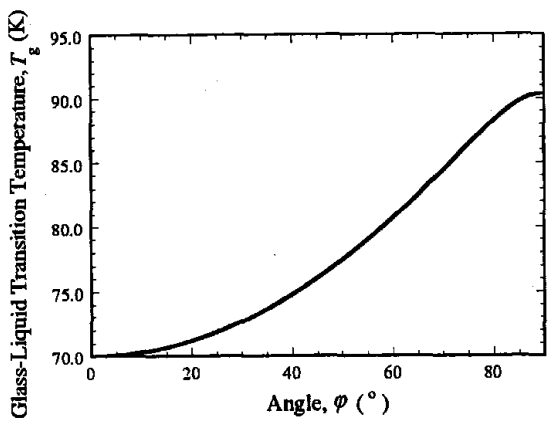

Fig. 6. $T_{g}$ vs. $\varphi$ characteristics at $B=1 \mathrm{~T}$ obtained from (11). $B_{\mathrm{g}, \perp}(0)=5.9 \mathrm{~T}, T_{\mathrm{c}}=101 \mathrm{~K}$, and $\beta=1.5$, respectively.

determined by (2). As can be seen, voltage traces vary drastically depending upon the field direction after turning on the heater. Namely, we can't see any dissipations for the properties at $\varphi=30,45$ and $60^{\circ}$ within the voltage window of Fig. 7 . Temperature increase is also small correspondingly. However, voltage and temperature increase rapidly for $\varphi=0,10$ and $20^{\circ}$. Fig. 9 shows the current sharing rate and the normalized temperature $\left(T / T_{\mathrm{g}}\right)$ at $t=16.0 \mathrm{~s}$, plotted as a function of angle. As can be seen, current sharing rate increases steeply when $T$ is higher than $T_{\mathrm{g}}$. Because

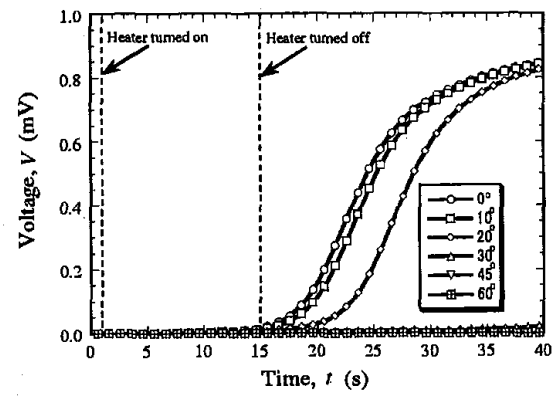

Fig. 7. Voltage traces for different angles with a transport current of $15.0 \mathrm{~A}$. Heater(heat flux of $1.8 \times 10^{5} \mathrm{Wm}^{-2}$ ) is turned on from $t=1.0$ to $15 \mathrm{~s}$. Initial temperature is $70.0 \mathrm{~K}$.

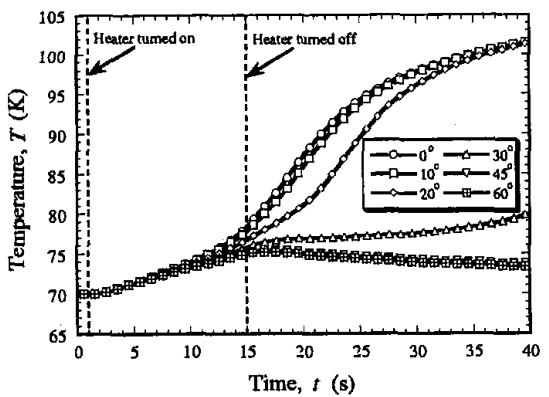

Fig. 8. Temperature traces for different angles. Heater(heat flux of $1.8 \times 10^{5} \mathrm{Wm}^{-2}$ ) is turned on from $t=1.0$ to $15 \mathrm{~s}$. Distance from the heater is $6.0 \mathrm{~mm}$. Initial temperature is $70.0 \mathrm{~K}$.

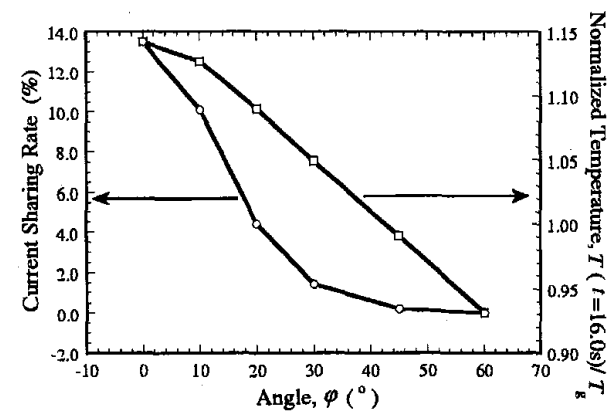

Fig. 9. Current sharing rate and normalized temperature $\left(T / T_{\mathrm{g}}\right)$ vs. angle $(\varphi)$ characteristics at $t=16.0$ s.

$E-J_{\text {sc }}$ curves are determined by $(3)$ at $T>T_{\mathrm{g}}$ and the dissipation is increased. That is to say, normal zone propagation properties depend on the field direction and stability have to be discussed by taking into account the field direction. Experimental study has been planned.

\section{CONCLUSION}

Normal propagation properties were studied in zero and high magnetic field based on the computer simulation. In zero magnetic field, the power-law expression for the $E-J$ curves was used to calculate the Joule heating. In high magnetic field, however, $E-J$ curves vary complicatedly, and the analytical expressions proposed by Kiss et al. was adopted. Anisotopy of the current transport characteristics were also considered. It was shown that the normal propagation property was influenced by the complicated $E-J$ characteristics and the anisotropy.

\section{ACKNOWLEDGMENT}

The authors would like to acknowledge Prof. Yamaguchi at Niigata university in Japan for providing samples.

\section{REFERENCES}

[1] S. Shimizu, A. Ishiyama, O. Kasuu, H. Ii, K. Takeda and M. Shibuya, "Normal-Zone Propagation Properties in BSCCO Silver Sheathed Tapes", Proceedings of the IEE of Japan, ASC-99-27, pp. $13-18,1999$ (in Japanese).

[2] T. Kiss, T. Nakamura, K. Hasegawa, M. Inoue, H. Okamoto, K. Funaki, M. Takeo, K. Yamafuji and F. Irie, "Nonlinear Resistance in High $T_{\mathrm{c}}$ Superconductors as a Function of Bias Current Density, Temperature and Magnetic Field", Proceedings of 17th International Cryogenic Engineering Conference, pp. 427-430, 1998.

[3] T. Kiss, T. Nakamura, N. Mishiro, K. Hasegawa, M. Inoue, M. Takeo, F. Irie and K. Yamafuji, "Transport Characteristics in High $T_{c}$ Superconductors", Proceedings of 15 th International Conference on Magnet Technology, part 2, pp. 1052-1055, 1998.

[4] H. Okamoto, F. Irie, T. Kiss, T. Nakamura and T. Kanazawa, " Characterization of $\mathrm{Ag} / \mathrm{Bi}-2223$ Tapes in Wide Range of Temperature and Magnetic Field", Proceedings of 15th International Conference on Magnet Technology, part 2, pp. 1071-1074, 1998.

[5] T. Nakamura, T. Yamamoto, S. Tsuchiya, A. Fujio, T. Hoshino, I. Muta, S. S. Oh and H. S. Ha, "Anisotropy of critical current and glassliquid transition flux density in B-2223/Ag tape" , Superconductor Science and Technology, vol.13, pp.1521-1525, 2000.

[6] S. B. Kim, A. Ishiyama, H. Okada and S. Nomura, "Normal-zone propagation properties in $\mathrm{Bi}-2223 / \mathrm{Ag}$ superconducting multifilamentary tapes ", Cryogenics, vol.38, pp.823-831, 1998. 